\title{
Die Medizin des 21. Jahrhunderts braucht eine neue Identität und neue Prioritäten in der Forschung
}

\section{Johannes Bircher}

Es bestehen keinerlei Interessenkonflikte. Es sind keine Firmen involviert mit Ausnahme des Verlages der Referenz [2], der natürlich gerne Bücher verkaufen würde.
Korrespondenz:

Prof. Dr. med. Johannes Bircher Reuelweg 20

$\mathrm{CH}-3045$ Meikirch

jbi@swissonline.ch

\section{Wo brennt es in der Medizin heute?}

Die Medizin ist ein grossartiges kulturelles Erbe der Menschheit. Wissenschaftliche und technologische Fortschritte haben sie aber in den letzten Jahrzehnten in eine schwierige Krise hineinmanövriert. Auf der einen Seite kann die Medizin heute soviel wie noch nie in der Geschichte, doch auf der anderen Seite ist ihre Komplexität derart gestiegen, dass sie nicht mehr sachgerecht gehandhabt werden kann. Unter den Krisensymptomen ist zunächst die Kostensteigerung besonders auffällig. In allen entwickelten Ländern ist sie höher als das Wachstum des Bruttosozialproduktes und damit nicht zukunftsfähig. Die meisten Kostensenkungsprogramme haben die Komplexität der Medizin weiter erhöht. Die Einführung marktwirtschaftlicher Strukturen ist mit bedeutenden Risiken von unerwünschten Wirkungen behaftet. $\mathrm{Zu}$ ihrer Kontrolle wurden Qualitätssicherungsprogramme eingeführt, deren Nutzen in der Medizin meist nicht wissenschaftlich nachgewiesen ist. Vermehrte Bürokratisierung und Reorganisationen beanspruchen von Ärzten und Pflegekräften viel Zeit, die den Patienten verlorengeht und das medizinische Personal demotiviert. Dazu kommt Angst vor Haftpflichtprozessen. Hierarchische Strukturen und autoritäre Verhaltensmuster sind der Komplexität der heutigen Medizin auch nicht mehr angemessen. Das Sozialprestige der Mediziner ist zwar immer noch höher als dasjenige aller anderen Berufsgruppen, aber dennoch haben Ärzte ihre Bestimmungsmacht über die Medizin verloren und können für ihre Patienten nur noch das tun, was bezahlt wird, und nicht mehr, was sie als richtig und angemessen erachten. Es ist deshalb nicht verwunderlich, dass Hingabe von Ärzten und Pflegenden selten, hingegen Burn-outPhänomene häufig geworden sind. Die aufgezeigten Entwicklungen sind nur die Spitze des Eisberges. Eine genauere Beschreibung müsste viel ausführlicher sein. Entscheidend ist die Schlussfolgerung, dass heute für das Wohl der Patienten nicht mehr das medizinisch Sinnvolle, sondern nur noch das Bezahlbare getan werden
La médecine du $X X I^{e}$ siècle

a besoin d'une nouvelle identité

\section{et de nouvelles priorités}

\section{dans la recherche}

La première raison des difficultés auxquelles est confrontée la médecine d'aujourd'hui est qu'elle n'est pas en mesure de véhiculer une représentation unique et fiable de la santé et de la maladie. C'est la raison pour laquelle la médecine ne peut communiquer de manière effective, comme peuvent le faire $d^{\prime}$ autres systèmes sociétaux, ses revendications propres. Nous devons dès lors nous orienter vers une nouvelle définition de la santé qui permette de mieux définir la médecine pour mieux la défendre. Le deuxième problème est que les modifications d'organisation en médecine, au contraire p.ex. de l'introduction des médicaments, ne sont pas fondées sur l'évidence scientifique. II faudrait pour cela une approche interdisciplinaire impliquant les domaines des sciences naturelles et humaines. A l'avenir, il faudra mettre en place des mesures d'organisation qui s'appuient elles aussi sur une médecine fondée sur des données probantes, ce qu'on appelle la «New EBM».

kann. In der Medizin ist nicht mehr Wissen und Können, sondern das Geld zum beschränkenden Faktor geworden. Entsprechend wird das Potential der Medizin nicht mehr voll genutzt, und es ist unredlich, der Öffentlichkeit jeden kleinen wissenschaftlichen Fortschritt lautstark anzupreisen, was von den Medien, der pharmazeutischen Industrie und sogar von Universitäten immer noch praktiziert wird. Dient eine solche Wissenschaft wirklich der Medizin, oder verfolgt sie andere Ziele? 
Bei sorgfältiger Betrachtung sind alle aufgezählten Trends und Entwicklungen bestens verständlich, resultieren sie doch aus Eigengesetzlichkeiten verschiedener gesellschaftlicher Systeme, die sich durch die Umstände gewissermassen von selbst ergeben [1]. Alle beteiligten Personen und Institutionen handeln nach ihrem besten Wissen und Gewissen, und offensichtlich trägt niemand die Schuld für die Misere. Wie aber ist so etwas möglich? Ich vertrete die Hypothese, dass die Medizin deshalb einen grossen Teil ihrer Selbstbestimmungsmacht eingebüsst hat, weil sie als System immer noch eine Identität vertritt, die dem 19. und beginnenden 20. Jahrhundert entspricht [2]. Damals waren sowohl Ärzte wie Pflegende wegen Infektionskrankheiten am Leben gefährdet und wurden entsprechend hoch geachtet. Mein Vater z. B. hat während des Studiums Freunde an Kinderlähmung und an Tuberkulose verloren. Damals hatten die Ärzte auch ein Wissensmonopol, und es galt, was sie sagten. Ihre Urteilsbildung wurde wenig hinterfragt. Heute riskieren Ärzte und Pflegefachkräfte wegen ihres Berufes nicht mehr ihr Leben. Zudem ist Wissen im Zeitalter des Internets allgemein verfügbar geworden und nicht mehr eine Exklusivität von Berufsgruppen. Klar ist auch, dass Urteilsbildung von den Perspektiven abhängt, aus denen ein Problem angeschaut und analysiert wird. Entsprechend haben sich Vertreter anderer gesellschaftlicher
Systeme medizinisches Wissen und eine gewisse Urteilsfähigkeit angeeignet und bestimmen heute die Medizin gemäss ihren eigenen Kriterien. Diese Verhältnisse lassen sich in der Tabelle 1 analysieren.

Jedes gesellschaftliche System stillt einen Bedarf, verfügt über Ressourcen, und seine Akteure handeln entsprechend dem dazugehörenden symbolischen Steuerungsmedium [1]. So besteht z.B. im Wirtschaftssystem ein Bedarf für Güter und Dienstleistungen. Als Ressource dient die Kaufkraft der Individuen. Die Handlungen bestehen im Kaufen oder Nichtkaufen, und alle Individuen sind diesbezüglich frei. Ob ein Kauf zustande kommt, wird deshalb - vereinfachend gesagt - in jedem einzelnen Fall in erster Linie durch das Geld geregelt. Denn auch wenn ein Bedarf besteht und die Ressourcen vorhanden sind, bleibt der Preis das entscheidende Kriterium dafür, ob ein bestimmtes Produkt oder eine bestimmte Dienstleistung gekauft wird oder nicht. Somit funktioniert im Wirtschaftssystem der Preis oder das Geld als symbolisches Steuerungsmedium. Der Sinn dieser Verhältnisse wird klar, wenn die hohe Effizienz des Wirtschaftssystems bei Respektierung der individuellen Freiheit bedacht wird. Grundsätzlich hat sich in der Wirtschaft «Marktverhalten» mit Geld als symbolischem Steuerungsmedium als hocheffizient erwiesen. Aus der Tabelle 1 wird ersichtlich, dass analoge Verhältnisse auch für andere gesellschaft-

Tabelle 1

Schematische Charakterisierung einiger gesellschaftlicher Systeme, um sie mit der Individualmedizin zu vergleichen (in Anlehnung an [1]).

\begin{tabular}{|c|c|c|c|c|c|}
\hline System & Bedarf & Ressourcen & Handlung & $\begin{array}{l}\text { Spezifisches } \\
\text { Steuerungsmedium* }\end{array}$ & Emergenz $^{* *}$ \\
\hline Wirtschaft & Güter, Dienstleistungen & Kaufkraft & Kaufen oder nicht kaufen & Geld in $€, \$, £$ bzw. Preis & $\begin{array}{l}\text { Optimaler Einsatz } \\
\text { der Ressourcen, Effizienz }\end{array}$ \\
\hline Politik & $\begin{array}{l}\text { Gesellschaftliche } \\
\text { Bestimmungsmacht }\end{array}$ & $\begin{array}{l}\text { Gesellschaftliche } \\
\text { Verantwortung, Machttrieb }\end{array}$ & Wahlen, Abstimmungen & Regeln der Demokratie & $\begin{array}{l}\text { Reduktion } \\
\text { von Machtmissbrauch }\end{array}$ \\
\hline Recht & Gerechtigkeit & Gerechtigkeitsbedürfnis & $\begin{array}{l}\text { Vereinbarung, } \\
\text { Gerichtsbeschluss }\end{array}$ & Gesetz & $\begin{array}{l}\text { Reduktion und } \\
\text { Lösung von Konflikten }\end{array}$ \\
\hline Verwaltung & Privilegien & Wunsch nach Privilegien & $\begin{array}{l}\text { Zusprache } \\
\text { oder Verweigerung }\end{array}$ & Verwaltungsordnung & Gleiche Regeln für alle \\
\hline Verkehr & Ortsveränderung & $\begin{array}{l}\text { Persönlicher Gewinn } \\
\text { an anderen Orten }\end{array}$ & $\begin{array}{l}\text { Privater } \\
\text { und öffentlicher Verkehr }\end{array}$ & $\begin{array}{l}\text { Verkehrsregeln, } \\
\text { Fahrplan, Flugplan }\end{array}$ & $\begin{array}{l}\text { Effizienz der } \\
\text { Ortsveränderung }\end{array}$ \\
\hline Wissenschaft & Neues Wissen & Neugier, Reputation & Finanzierung, Publikation & Peer review & $\begin{array}{l}\text { Optimierter Einsatz } \\
\text { der Mittel }\end{array}$ \\
\hline Medizin & $\begin{array}{l}\text { Sicherung und } \\
\text { Verbesserung des } \\
\text { Gesundheitszustandes }\end{array}$ & $\begin{array}{l}\text { Wunsch nach Erhaltung } \\
\text { der Gesundheit, } \\
\text { Heilung und Linderung }\end{array}$ & $\begin{array}{l}\text { Prophylaxe, Diagnose } \\
\text { und Therapie }\end{array}$ & $\bullet$ & $\begin{array}{l}\text { Verlängerte Lebens- } \\
\text { erwartung in Gesundheit }\end{array}$ \\
\hline
\end{tabular}

* Das symbolische Steuerungsmedium entscheidet über und koordiniert die Beziehung zwischen Bedarf und Handlung, d.h., die Handlung wird durch das symbolische Steuerungsmedium gesteuert.

** Emergenz beschreibt den Gewinn eines Systems, an dem die Subsysteme als Teilhaber des Systems partizipieren können, wenn es wirklich durch das symbolische Steuerungsmedium geregelt wird. 
liche Systeme beschrieben werden können. In jedem von ihnen besteht ein symbolisches Steuerungsmedium, das für das entsprechende System spezifisch ist. Aus diesen Überlegungen ergibt sich die Frage, welches das spezifische symbolische Steuerungsmedium für die Medizin sein könnte. Und diese Frage ist zurzeit nicht leicht zu beantworten.

Wird die Medizin nämlich in erster Linie durch Geld gesteuert, so verfügt sie über keine eigene Identität mehr, sondern wird Teil des Wirtschaftssystems. Natürlich werden immer noch Diagnosen gestellt und Therapien durchgeführt, doch die Patienten werden zu Kunden, und nichts Medizinspezifisches unterscheidet medizinische Dienstleistungen von anderen Handlungen im Rahmen des Wirtschaftssystems. Oder wenn die Verwaltung die Medizin regelt, wird sie Teil des Verwaltungsapparates. Diese Beispiele weisen wieder darauf hin, dass das Spezifische eines Systems und seine Identität eng mit dem symbolischen Steuerungsmedium verbunden sind. Deshalb besteht die logische Konsequenz darin, dass die Medizin als eigenständiges gesellschaftliches System ihr Potential so lange nicht realisieren kann, als sie nicht über ein medizinspezifisches symbolisches Steuerungsmedium verfügt. Offensichtlich fehlt ihr das zurzeit. Diese Betrachtung erklärt somit, weshalb die Medizin heute von allen Seiten durch verschiedene gesellschaftliche Systeme bedrängt wird und sich dabei nicht eigenständig und sachgerecht behauptet. Das ist für Patienten ein Verlust, für die Medizin schädlich und für medizinisches Personal frustrierend.

\section{Was könnte ein medizinspezifisches symbolisches Steuerungsmedium sein?}

Spezifisch für die Medizin ist, dass sie sich vorwiegend darum bemüht, Krankheiten vorzubeugen und bei kranken Menschen den Gesundheitszustand $\mathrm{zu}$ verbessern. Im besten Fall bedeutet dies Heilung, aber wenn dies nicht möglich ist, stehen heute viele Möglichkeiten der Linderung zur Verfügung. Todkranke Menschen werden auf ihrem letzten Weg medizinisch begleitet. Daraus folgt, dass als symbolisches Steuerungsmedium in erster Linie eine zeitgemässe und sachgerechte Vorstellung von Gesundheit und Krankheit in Frage kommt [2]. Diese Idee ist allerdings dadurch kompliziert, dass es sowohl in der Medizin als auch in der Gesellschaft keinen Konsens darüber gibt, mit welchen Inhalten die Begriffe Gesundheit und Krankheit zu füllen sind. Ein kürzlich publiziertes Beispiel ist im Kasten zusammengefasst [3]. Es gehört zwar zu einer guten akademischen Tra- dition, Probleme unter verschiedenen Gesichtspunkten zu betrachten, doch dient diese Denkweise der Erarbeitung eines symbolischen Steuerungsmediums kaum, denn dafür ist nur eine Gesundheitsdefinition geeignet, auf die sich Angehörige der Medizin und Vertreter der Gesellschaft geeinigt haben und sich gemeinsam stützen wollen.

Eine solche Definition gibt es zurzeit nicht, obwohl viele an sich wertvolle Beschreibungen von Gesundheit und Krankheit zirkulieren [4]. Ihre Besprechung würde den Rahmen dieser Ausführungen sprengen. Als Beispiel möchte ich nur auf die Formulierung von Lennart Nordenfelt kurz eingehen, denn sie besticht durch ihre Prägnanz und Kürze: «Eine Person ist völlig gesund, wenn und nur wenn sie unter Standardbedingungen die Fähigkeit hat, alle ihre vitalen Ziele zu verwirklichen» $[5,6]$. Bei dieser Festlegung ist leider der Abstraktionsgrad derart hoch, dass ihre Anwendung in der Praxis schwierig ist. Zwar diskutiert Nordenfelt die Begriffe der Standardbedingungen und der vitalen Ziele sehr sorgfältig, doch sind sie für den medizinischen Alltag schwierig ein- und umzusetzen. Aus diesen Gründen habe ich als Basis für einen zukünftigen Diskurs eine neue Definition der Gesundheit vorgeschlagen $[2,7]$.

Im Meikirch-Modell ist «Gesundheit ein dynamischer Zustand von Wohlbefinden, bestehend aus einem biopsychosozialen Potential, das genügt, um die alters- und kulturspezifischen Anforderungen des Lebens in Eigenverantwortung zu befriedigen. Krankheit ist der Zustand, bei dem das Potential diesen Anforderungen nicht genügt» [2]. Das Besondere dieser Formulierung besteht darin, dass es grundsätzlich zur Praxis einer guten Medizin gehört, alle ihre Komponenten - die harten wie die weichen - in einer ärztlichen Untersuchung einzeln zu prüfen und zu beurteilen. Dabei besteht das Potential aus zwei Komponenten, nämlich einer biologisch gegebenen und einer persönlich erworbenen (Abb. 1).

Das biologisch gegebene Potential resultiert zunächst aus der genetischen Ausrüstung und aus der Qualität der Schwangerschaft. Bei der Geburt hat es einen von Mensch zu Mensch verschiedenen, endlichen Wert und nimmt anschliessend bei jeder weiteren Prägung ab. Im Verlauf des Lebens kann es durch akute oder chronische Krankheiten oder durch Unfälle Einbrüche erleiden. Bei angemessener Sorgfalt bleibt es länger verfügbar als im Falle von Raubbau. Zum Zeitpunkt des Todes beträgt es null. Das persönlich erworbene Potential ist bei der Geburt noch klein, nimmt zunächst natürlicherweise rasch zu, wird 


\section{Abbildung 1}

Potential eines Menschen als Funktion der Lebenszeit im Meikirch-Modell. Das Potential besteht aus zwei Komponenten. Das biologisch gegebene Potential ist zum Zeitpunkt der Geburt am grössten, nimmt anschliessend nur noch ab und beträgt beim Tod null. Das persönlich erworbene Potential ist bei der Geburt klein, nimmt dann zunächst rasch und später langsam zu und kann das ganze Leben lang ansteigen, sofern sich ein Individuum darum bemüht. Die Summe der beiden Potentiale ist das Gesamtpotential, das für die Gesundheit zur Verfügung steht. Die Kurven sind idealisiert gezeichnet. In Wirklichkeit schwanken sie und sind individuell sehr verschieden (Details s. [2]).

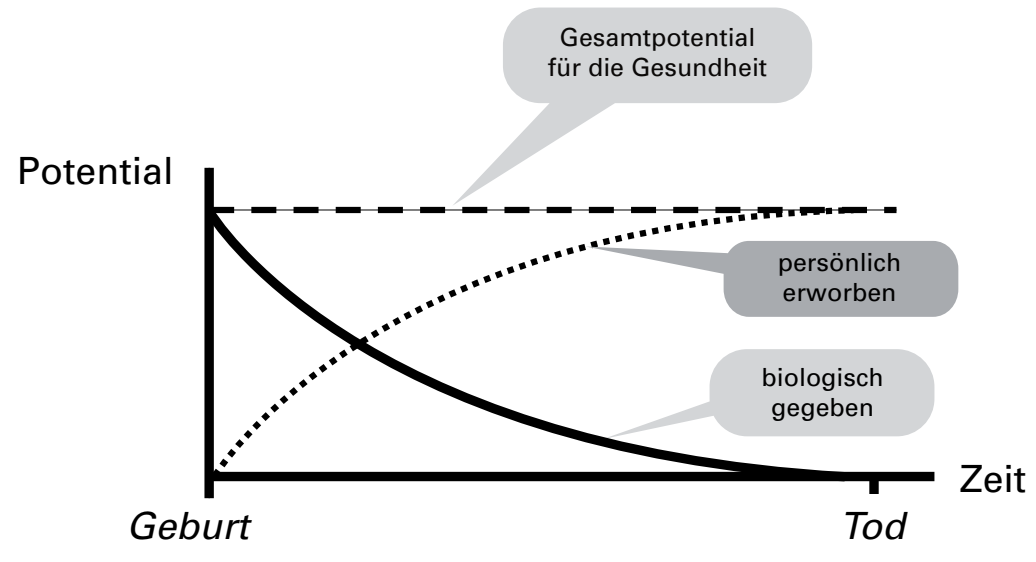

aber mit der Zeit immer mehr von der Lebensführung der betreffenden Person bestimmt. Es kann das ganze Leben lang ansteigen, sofern sich ein Mensch darum bemüht. Dabei umfasst es nicht nur alles emotional und kognitiv Gelernte, sondern auch somatische Aspekte wie etwa einen trainierten Körperbau oder Immunität gegenüber durchgemachten Krankheiten. Auch die Qualität des sozialen Netzes gehört dazu. Die Konzepte der Salutogenese von Antonowsky sind auf das persönlich erworbene Potential anwendbar [8]. Auch wenn in der Abbildung 1 die Ordinate nicht numerisch ist, muss man sich vorstellen, dass die Summe der beiden Potentiale das Gesamtpotential darstellt, das für die Gesundheit zur Verfügung steht. Die Kurven sind idealisiert gezeichnet. In Wirklichkeit verlaufen sie unregelmässig und unterscheiden sich von Mensch zu Mensch.

Bevor eine neue Gesundheitsdefinition zum symbolischen Steuerungsmedium für die Medizin werden kann, muss sie in einem gesellschaftlichen Prozess von den relevanten Verantwortungsträgern beraten und akzeptiert werden. Dies hat im Falle des Meikirch-Modells bisher noch nicht stattgefunden. Die Diskussion dazu ist aber angestossen. Bei Akzeptanz wird die Medizin dadurch effektiver, dass sie die beiden Teilpotentiale ihrer Patienten und die anderen Komponenten des Meikirch-Modells ausgewogener als bisher berücksichtigt. Für die Erschliessung des vollen Potentials der Medizin ist zudem die Tatsache entscheidend, dass eine Gesundheitsdefinition der bisher fehlende Bestandteil einer Beschreibung der Identität der Medizin ist [2]. Das Meikirch-Modell kann diese Lücke füllen (Abb. 2).

\section{Integration einer wiedererstarkten Medizin in die Gesellschaft}

Auch wenn sich die Medizin dank einer neugewonnenen Identität wieder als gesellschaftliches System gegenüber anderen Systemen behaupten kann, bedeutet das nicht, dass sie sich unbehindert entfalten darf. Aus systemtheoretischer Sicht muss sie begreifen, dass sie Teil des übergeordneten Systems der Gesellschaft ist und ihren Beitrag zu deren Funktion zu leisten hat. Um das zu erläutern, ist es nötig, die Beziehung von Subsystemen zu ihrem übergeordneten System ganz allgemein zu beleuchten. Das lässt sich zunächst anschaulich am menschlichen Organismus illustrieren, denn er ist aus Organsystemen zusammengesetzt. Dabei wird die Tatsache evident, dass eine Person mehr ist als die Summe ihrer Organsysteme. Dieses «Mehr» wird mit dem Begriff Emergenz beschrieben [1]. Wenn die einzelnen Subsysteme - hier z. B. die Organsysteme im Sinne des Ganzen gut und koordiniert zusammenarbeiten, ist die Emergenz gross. Sie reduziert sich, wenn aus irgendeinem Grund z.B. wegen einer Krankheit - die Koordination nicht gut ist. Prototyp einer solchen Entwicklung ist das Wachstum eines Malignoms, dessen unkoordiniertes Verhalten einen Organismus sogar zum Erliegen bringen kann.

Nun bedeutet aber Eingliederung in ein grösseres Ganzes, dass die einzelnen Teile, d.h. die Subsysteme, Freiheitsgrade aufgeben müssen, und es stellt sich die Frage, weshalb es denn sinnvoll sein soll, freiwillig seine Freiheitsgrade einzuschränken. Die Antwort fällt deutlich aus. Partizipation an der Emergenz des übergeordneten Systems kompensiert nicht nur den Verlust an eigenen Freiheitsgraden der einzelnen Subsysteme, sondern verschafft ihnen einen zusätzlichen Gewinn [1]. Dieser Zusammenhang lässt sich besonders eindrücklich am Strassenverkehr aufzeigen. Zu den «einschränkenden» Verkehrsregeln gehören das Fahren auf der rechten Seite der Strasse, der Rechtsvortritt und der Vortritt im Kreisverkehr. Wenn sie nicht wären, müssten Autofahrer bei jeder Begegnung mit einem anderen Auto zunächst miteinander aushandeln, ob sie sich rechts oder links kreuzen wollen usw. Die Effizienz des Strassenverkehrs, d.h. seine Emergenz, wäre verschwindend klein. Diese Tatsache ist für alle Verkehrsteilnehmer leicht wahrnehmbar. Deshalb werden die angesprochenen 


\section{Abbildung 2}

Die Medizin ist ein Dienst am Menschen, dessen Identität vier Seiten aufweist. Es sind die Ziele, die Vorstellungen von Gesundheit, die Professionalität und die Orientierung [2]. Die Ziele sind in einem internationalen Projekt des Hastings Center erarbeitet worden [9]. Die Professionalität ist in einer Charta festgehalten [10]. Die medizinische Ethik wird heute durch Kommissionen und Ethikberater eingebracht. Eine Definition der Gesundheit hat bisher gefehlt.

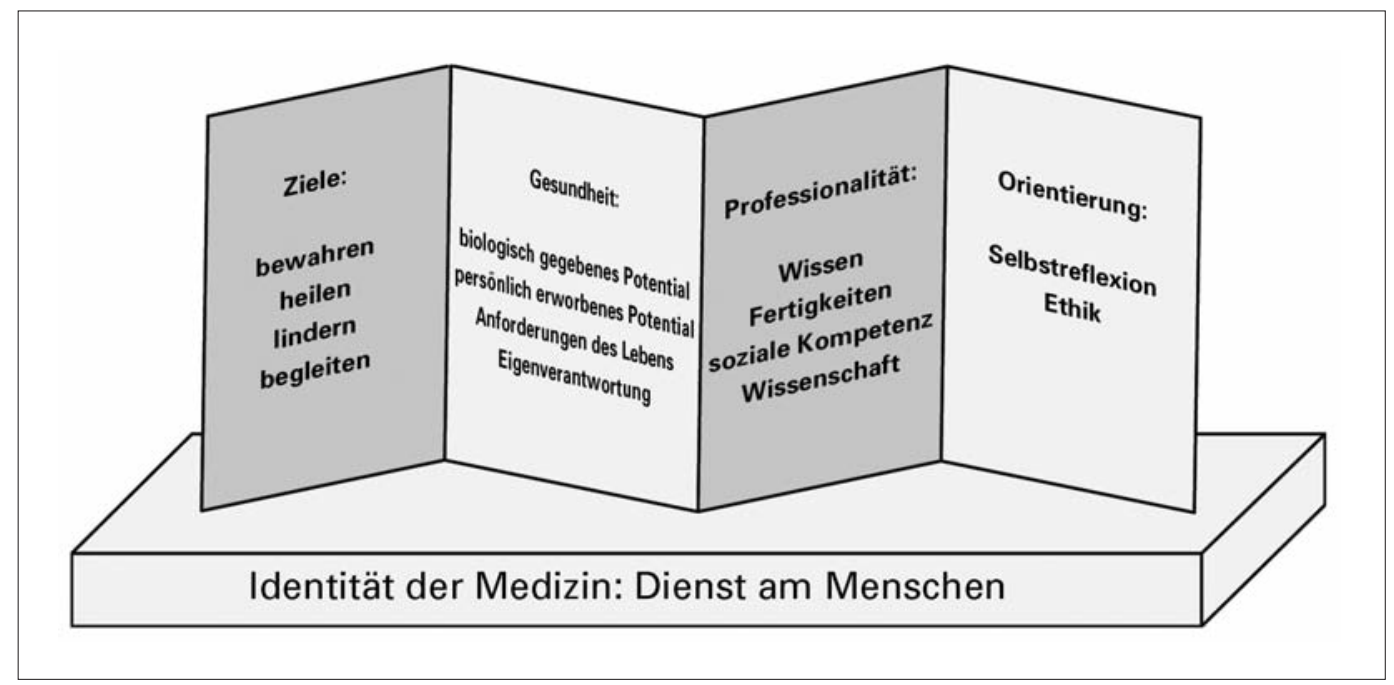

Grundregeln des Strassenverkehrs ohne weiteres eingehalten. Wenn es aber darum geht, z. B. vorgeschriebene Geschwindigkeiten nicht zu überschreiten, erleben die Verkehrsteilnehmer nur wenig oder keine Emergenz. Darum werden Geschwindigkeitsbeschränkungen oft missachtet, und selbst Bussen genügen nicht, sie durchzusetzen. Dieses Beispiel zeigt, dass ein selbstbestimmter Verzicht auf Freiheitsgrade die Einsicht

Vier kürzlich publizierte Gesundheitskonzepte, die die Schwierigkeit der Medizin illustrieren, sich auf ein einheitliches Konzept zu einigen [3].

\section{Der biomedizinische Ansatz}

Die Laborergebnisse und funktionellen Tests entscheiden darüber, ob der Patient gesund oder krank ist. Diese Sicht der Dinge ist quasi-offiziell (d. h. gesetzlich verankert und daher auch von den Versicherungen anerkannt).

\section{Der funktionelle Ansatz}

Gesundheit und Krankheit werden nach ihrer gesellschaftlichen Bedeutung beurteilt. Gesund ist demnach derjenige, der seine soziale Rolle voll ausfüllt und sich daher auch an sich ändernde Bedingungen anpassen kann.

\section{Der idealistische Ansatz}

Leitgedanke ist das allgemeine Wohlbefinden des einzelnen (in der Definition der WHO). Das Gleichgewicht zwischen allen Faktoren (physischen, emotionalen, sozialen, spirituellen und mentalen) begünstigt die Verwirklichung der Lebensziele.

\section{Der subjektive Ansatz}

Jeder einzelne hat seine eigene Vorstellung davon, was Gesund- und was Kranksein bedeutet. Die Gesundheit wird dabei als ein Energiekapital betrachtet, das es dem einzelnen ermöglicht, die Dinge zu verwirklichen, die in seinem Leben wichtig erscheinen. in den Wert der Emergenz voraussetzt. Deshalb wird sich die Medizin am erfolgreichsten in die Gesellschaft integrieren können, wenn sich alle Verantwortungsträger darum bemühen, Gewinne durch eine gute Zusammenarbeit, d.h. durch die Emergenz, für alle Betroffenen deutlich sichtbar und erlebbar zu machen. Das ist keine leichte Aufgabe.

Zurzeit wird in vielen Bereichen die Beziehung zwischen der Medizin und den anderen gesellschaftlichen Systemen neu ausgehandelt. Das ist an sich eine ideale Ausgangsbasis für die Medizin, um eine neugewonnene Identität zur Geltung zu bringen. Das Bewusstsein ihrer Identität ist aber nur die erste Voraussetzung für Erfolg. Die zweite und ebenso essentielle Vorbedingung besteht darin, dass die Angehörigen der Medizin und anderer gesellschaftlicher Systeme von der Emergenz einer neuen Ordnung überzeugt werden können. Wie beim Strassenverkehr funktioniert das viel eher, wenn jeder und jede einzelne den Gewinn einsehen kann, den er oder sie durch die spezifische Art der Integration der Medizin in die Gesellschaft realisieren kann. Eine überzeugende wissenschaftliche Evaluation aller neuen Organisationsformen ist deshalb von allergrösster Wichtigkeit und Dringlichkeit.

Diese Forderung wird dadurch unterstützt, dass die Beziehung zwischen der Medizin und dem Gesundheitssystem in der Ärzteschaft eher gemäss einer historischen als einer systemtheoretischen Rationalität betrachtet wird. So hat kürzlich eine Gruppe von Expertinnen und Experten der Schweizerischen Akademie der Medizini- 
schen Wissenschaften die Medizin und das Gesundheitswesen überlappend, gewissermassen «symbiotisch» dargestellt und die beiden Systemebenen nicht als qualitativ völlig verschieden auseinandergehalten [11]. Dadurch wird natürlich nicht klar, welche Entscheidungsmöglichkeiten, Abhängigkeiten und Verantwortlichkeiten der Medizin und welche dem Gesundheitssystem zugeordnet werden müssen. Deshalb besteht in der Ärzteschaft gegenüber Innovationen vorwiegend eine defensive Grundhaltung und ein bedeutendes Frustrationspotential. Diese Haltung wurde natürlich dadurch unterstützt, dass sich zahlreiche Innovationen als unwirksam oder sogar als schädlich erwiesen haben. Die Glaubwürdigkeit neuer organisatorischer Strukturen und Abläufe sowie neuer Kontroll- und Finanzierungsmethoden ist heute deutlich angeschlagen.

Vertrauen in Emergenz des Gesundheitssystems muss durch Sinnstiftung und wissenschaftliche Evidenz wiederhergestellt werden. Organisationsformen, die mit der Identität der Medizin kompatibel sind und diese stärken, haben gute Chancen, von allen interessierten Personen als grundsätzlich sinnvoll betrachtet zu werden. Heute genügt das allerdings nicht mehr. Erst sorgfältig erarbeitete wissenschaftliche Evidenz schafft Glaubwürdigkeit. Sie ist ein wichtiger und dringender Bedarf und fordert für die medizinische Forschungspolitik ein Überdenken ihrer Prioritäten. Die einseitige Fokussierung auf molekularbiologische Erkenntnisse ist nicht mehr zu verantworten. Eine Patientenbetreuung, die durch finanzielle Engpässe und nicht durch die Grenzen der Wissenschaft eingeschränkt wird, schreit geradezu nach einer neuen Forschungsstrategie. Die Medizin braucht einen bedeutenden interdisziplinären Schwerpunkt in der Versorgungsforschung im weitesten Sinne. Die Praxis der Medizin ist nicht nur medizinisch, sondern auch soziologisch, philosophisch, psychologisch, theologisch und natürlich auch wirtschaftswissenschaftlich zu untersuchen. In
Zukunft sollten organisatorische Innovationen nur noch implementiert werden dürfen, wenn sie sich auf wissenschaftliche Evidenz stützen können [12]. Sie werden auch nach ihrer Einführung nur so lange glaubhaft bleiben, als ihre Emergenz gezeigt werden kann. Eine neue Form von evidenzbasierter Medizin (New EBM) ist das Gebot der Stunde.

\section{Literatur}

1 Willke H. Systemtheorie. 3. Auflage. Stuttgart, New York: Gustav Fischer; 1991.

2 Bircher J, Wehkamp K-H. Das ungenutzte Potential der Medizin - Analyse von Gesundheit und Krankheit zu Beginn des 21. Jahrhunderts. Zürich: Rüffer und Rub; 2006.

3 Paccaud F. Prävention von Krankheiten und öffentliche Gesundheit. In: Kocher G, Oggier W. Gesundheitswesen Schweiz 2007-2009. Bern: Hans Huber; 2007. S. 279-90.

4 Franke A. Modelle von Gesundheit und Krankheit. Bern: Hans Huber; 2006.

5 Nordenfelt L. On the Nature of Health - An ActionTheoretic Approach. Dordrecht: Kluwer; 1995.

6 Nordenfelt L. On the Evolutionary Concept of Health: Health as Natural Function. In: Nordenfelt L, Liss P-E. Dimensions of Health and Health Promotion. Amsterdam, New York: Rodopi; 2003. S. 37-54.

7 Bircher J. Towards a dynamic definition of health and disease. Med Health Care Philos. 2005;8:335-41.

8 Antonovsky A. Salutogenese - Zur Entmystifizierung der Gesundheit. Tübingen: dgvt-Verlag; 1997.

9 The goals of medicine. Setting new priorities. Hastings Cent Rep. 1996;26(6):S1-27.

10 Medical professionalism in the new millennium: a physician charter. Ann Intern Med. 2002; 136:243-6.

11 Ziele und Aufgaben der Medizin zu Beginn des 21. Jahrhunderts. Bericht der ExpertInnengruppe der Schweizerischen Akademie der Medizinischen Wissenschaften. Basel: SAMW; 2004.

12 Auerbach AD, Landefeld CS, Shonjana K. The tension between needing to improve care and knowing how to do it. N Engl J Med. 2007;357: 608-13. 